\title{
Citas florísticas de la Sierra del Rincón y zonas limítrofes (Comunidad de Madrid)
}

\author{
Jorge Baonza Díaz ${ }^{1}$ Javier Herraiz Garrote² y Ángel Quirós Menéndez²
}

Resumen: Baonza Díaz, J.; Herraiz Garrote, J. \& Quirós Menéndez, A. 2015. Citas florísticas de la Sierra del Rincón y zonas limítrofes (Comunidad de Madrid). Bot. Complut. 39: 71-78.

Se aportan citas de flora vascular de interés en la Comunidad de Madrid localizadas en la Reserva de la Biosfera de la Sierra del Rincón y zonas limítrofes. En la discusión se destaca la presencia de flora atlántica y se señala como el cambio litológico de gneises a rocas sedimentarias menos metamorfizadas marca el cambio de matorrales de genisteas en la zona perteneciente al macizo de Somosierra a brezales en la zona ayllonense.

Palabras clave: flora atlántica, biogeografía, Sistema Central, España.

Abstract: Baonza Díaz, J.; Herraiz Garrote, J. \& Quirós Menéndez, A. 2015. Floristic notes of Sierra del Rincón Biosphere Reserve (Spain, Madrid province). Bot. Complut. 39: 71-78.

New records of vascular plants from Sierra del Rincón Biosphere Reserve and surroundings (Spain, Madrid province) are provided. It is noteworthy the presence of atlantic flora in this continental area and the different shrubby communities in different sectors with different litology: in areas with gneiss they are dominated by leguminous genisteae; where it is schistous, shale or quartzite they are heathlands.

Key words: atlantic flora, biogeography, Spanish Sistema Central range.

\section{INTRODUCCIÓN}

La Sierra del Rincón se ubica en el NE de la Comunidad de Madrid y ha sido declarada Reserva de la Biosfera en 2005. A pesar de los años transcurridos desde la declaración de este espacio protegido, y de ser la conservación y el apoyo a la investigación alguna de sus funciones declaradas, todavía carece de un estudio florístico completo, más allá de un simple listado de las especies de flora vascular presentes (Cuevas et al. 2005). Desde entonces ha habido algunas aportaciones puntuales nuevas (Baonza 2012, 2014; Enríquez de Salamanca 2009). Un estudio inconcluso eleva el catálogo florístico del espacio a cerca de 1000 taxones (Baonza 2012). Si la Reserva de la Biosfera carece de un estudio completo de su flora, el conocimiento de su entorno madrileño es casi anecdótico. Se muestran aquí algunas citas de interés de este amplio espacio necesitado de mejor conocimiento.

\section{MATERIALES Y MÉTODOS}

La Reserva de la Biosfera «Sierra del Rincón» y municipios limítrofes se corresponde a la comarca forestal IV denomi- nada «Montejo», según la división del territorio forestal de la Comunidad de Madrid establecido por Orden 4634/2006 (BOCM n ${ }^{\circ} 19$, de 23 de enero de 2007). Incluye los municipios de Horcajuelo de la Sierra, Montejo de la Sierra, La Hiruela, Prádena del Rincón y Puebla de la Sierra (dentro de la Reserva) y Puentes Viejas, Berzosa del Lozoya, Robledillo de la Jara, El Atazar y Cervera de Buitrago (Fig. 1).

Geográficamente es una sierra heterogénea, de reciente definición, que comprendería territorios de las tradicionalmente consideradas Sierra de Ayllón (en sentido amplio) y Somosierra, dentro del Sistema Central oriental. Geológicamente serían ayllonenses las sierras formadas por sedimentos paleozoicos ordovícicos y postordovícicos (principalmente esquistos, pizarras y cuarcitas) de La Puebla, La Hiruela y El Atazar, aquí con posición ESE. El resto del territorio, ocupando la zona al ONO, sobre gneises preordovícicos, pertenecería al macizo de Somosierra. Ambas zonas quedan separadas por la falla 0 cabalgamiento de Berzosa, que se extiende en dirección N-S desde las inmediaciones de Riaza en Segovia hasta Torrelaguna en Madrid (véase por ejemplo Vera et al. 2004, Muñoz \& Sanz 1995).

Las presentes citas proceden de observaciones directas de los autores en los últimos 10 años, de las que se han seleccionado aquellas citas que se consideran de interés, teniendo en cuenta su presencia conocida en la Comunidad de Madrid. A tal fin se han consultado las referencias en las bases de datos en in-

\footnotetext{
${ }^{1}$ Bustarviejo 28720 (Madrid), gejorbd@gmail.com

2 Casa Forestal «Las Gariñas», ctra. de Buitrago a Mangirón, pk 1,500; 28730 Buitrago del Lozoya (Madrid), jherraizg@hotmail.com

Recibido: 216 enero 2015. Aceptado: 6 febrero 2015.
} 

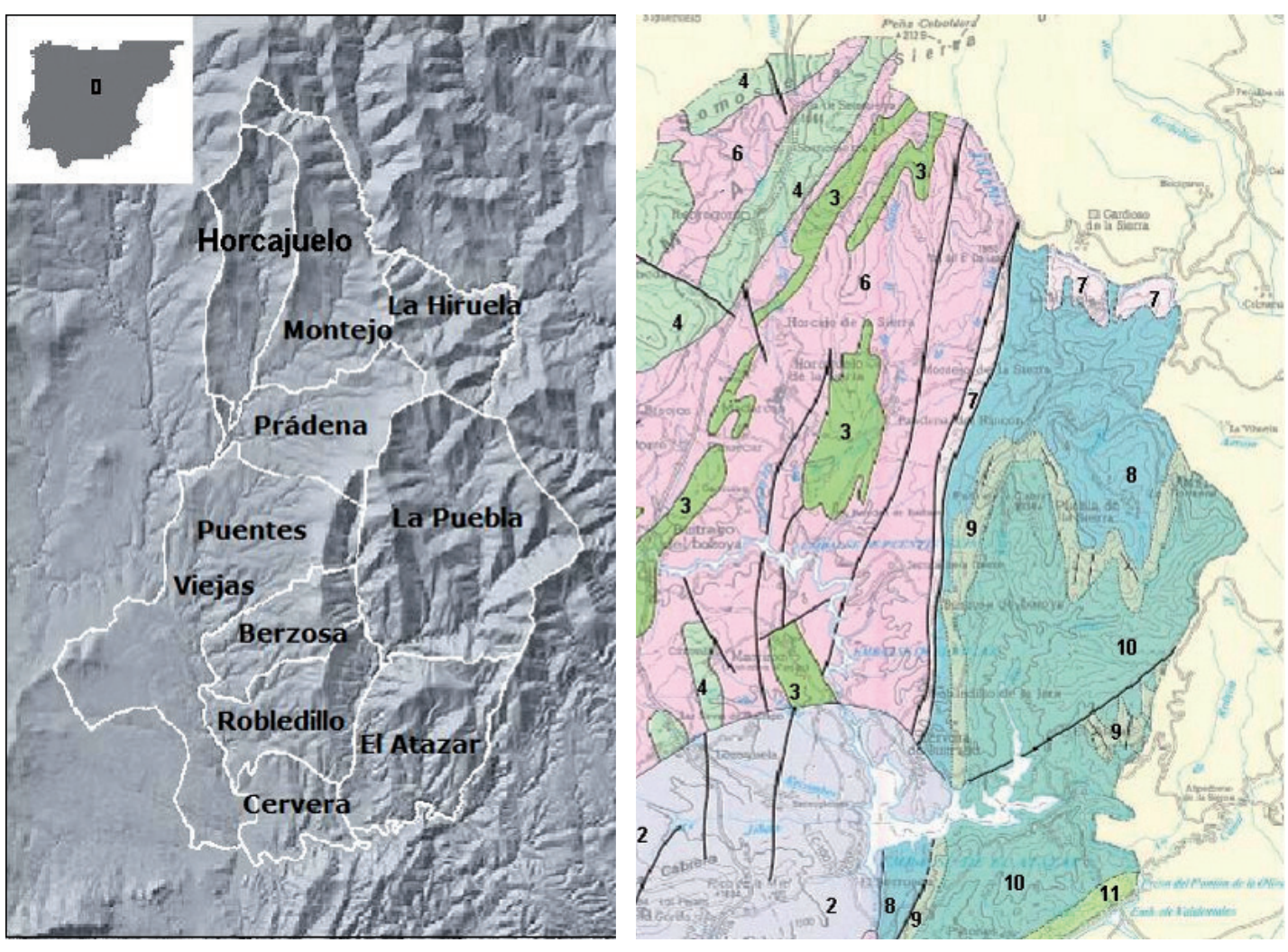

Fig. 1 - Izquierda: mapa de la comarca de Montejo. Derecha: mapa litológico del NE de la Comunidad de Madrid: 2 granito, 3 leucogneis no glandular metaplutónico, 4 ortogneis glandular metaplutónico, 6 gneis glandular y esquistoso con anfibolitas cámbrico-precámbrico, 7 gneis glandular cámbrico-precámbrico, 8 esquistos con intercalaciones de cuarcitas y anfibolitas ordovícicos, 9 cuarcita ordovícica, 10 pizarras y cuarcitas ordovícicas, 11 areniscas calizas y dolomías cretácicas (a partir del Atlas Geocientífico de Madrid (IGME 1988), modificado.

ternet (principalmente www.anthos.es) y otros trabajos no incluidos en la misma.

\section{RESULTADOS}

\section{Allium scorzonerifolium Desf. ex DC.}

M: Puebla de la Sierra, 30TVL6037, $1110 \mathrm{~m}$, ladera rocosa (Baonza 2-8-2011, v.v.). Puebla de la Sierra, Fuente Palomera, 30TVL6538, $1500 \mathrm{~m}$, juncal y brezales E. arborea (Baonza, 16-07-2011, MA 886536).

Confirma una cita del s. XIX en la zona: Puebla de la Mujer Muerta, barrancos, riberas y parajes más o menos húmedos, montañas y elevaciones ácidas (Cutanda s.f., MA 148973; Cutanda 1861: 657). Conocido de zonas cercanas en Guadalajara (Majaelrayo, Tamajón) y en Madrid de Rozas de Puerto Real, La Pedriza, Patones y El Atazar (revisión en Cebolla \& Rivas 1994, más algunos pliegos recopilados en anthos.es).

\section{Adenocarpus argyrophyllus (Rivas Goday) Caball.}

M: La Puebla, ladera O de Peña de la Cabra, en la cuenca del Arroyo del Matizo, 30TVL5939, pedregal y roquedos cuarcíticos con Erica arborea, abundante, 1350-1750 m en orientación sur y oeste (Herraiz, 15-11-2006, v.v.; Baonza \& Quirós, 24-05-2014, MA 886531). Ibídem, 30TVL5839, en laderas pedregosas a $1300 \mathrm{~m}$ en orientación NO, entre plantaciones de pinos (Baonza \& Quirós, 24-05-2014, v.v.).

Tercera y mayor población de esta interesante especie en La Puebla, donde se conocen otras dos poblaciones: 30TVL6044, a $1725 \mathrm{~m}$ en cresta cuarcítica y 30TVL6336, a 1120 m en ladera rocosa en umbría (Baonza 2012). En conjunto representan una notable disyunción corológica de las poblaciones gredenses o los Montes de Toledo co- 

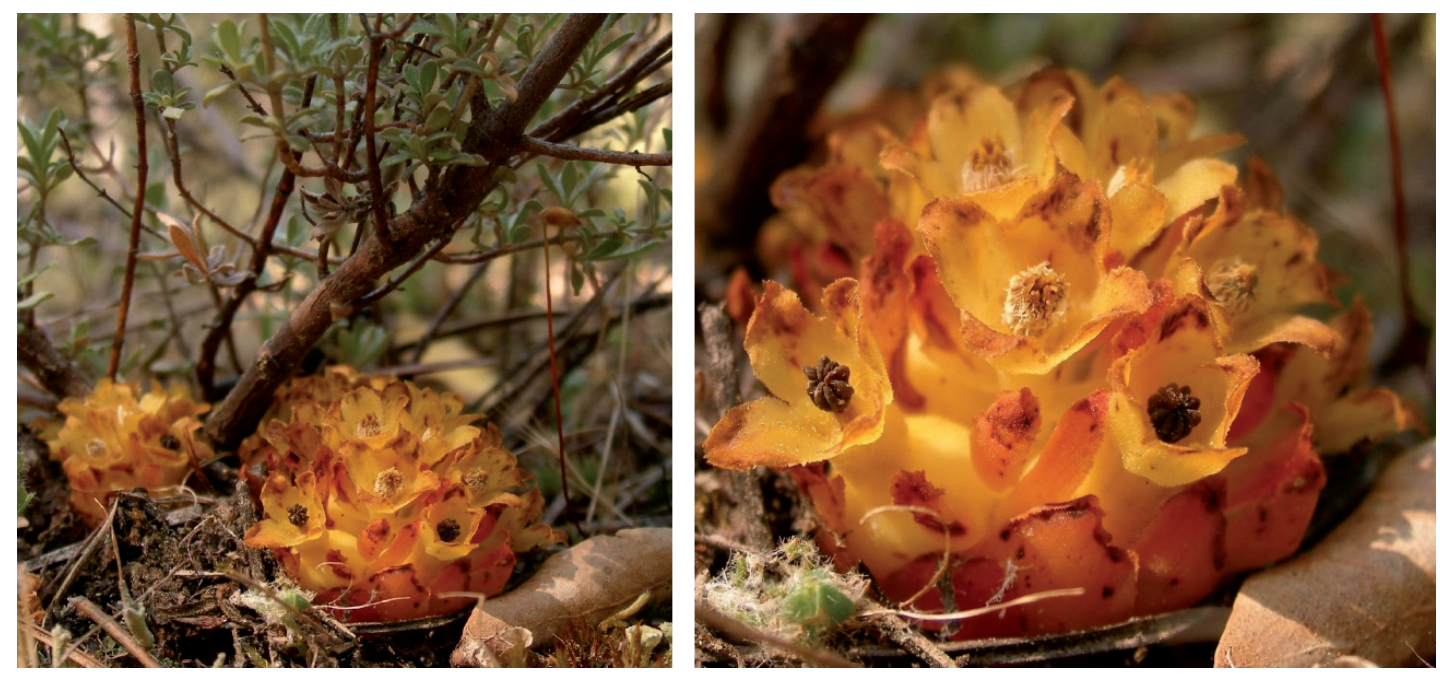

Fig. 2-Cytinus hypocistis subsp. macranthus Wettst. en Halimium ocymoides. Paredes de Buitrago-Gandullas, Monte de Utilidad Pública «Perímetro de Paredes» (Quirós 12-06-2014).

nocidas. A esta especie parecen corresponder realmente las observaciones sobre Adenocarpus hispanicus en $\mathrm{La}$ Puebla (López 2001).

\section{Chaetonychia cymosa (L.) Sweet.}

M: Puebla de la Sierra, bajo el Collado Grande, 30TVL5935, $1000 \mathrm{~m}$, pastizal terofítico en litosuelo de pizarras en claro de jaral pringoso (Baonza, 14-4-2011, MA 886526).

Planta muy poco citada en Madrid o las sierras de Guadarrama y Ayllón, la mayoría muy antiguas. M: Torrelodones, [30TVK29], en jaral (Rivas Goday 1958). La Moraleja tract. Matritens [30TVK48] (BC-Bernades 225, Ibáñez et al 2009). Miraflores [30TVL31] (Cutanda 1861: 303; Willkomm \& Lange 1874-1880). El Cuadrón, río Lozoya, 30TVL4335, $1030 \mathrm{~m}$, pastizales terofíticos silicícolas (Fernández 1988). Sg: Becerril, [30TVL67] (Mayor 1975).

\section{Cytinus hypocistis subsp. macranthus Wettst.}

M: El Atazar, cuencas de los Arroyos de Redubia y Refredillo, 30TVL6331, 1.100 m, pinar de repoblación de $P$. pinaster con sotobosque de jaral de C. ladanifer (Quirós, 28-04-2007, v.v.). Prádena del Rincón, Lomo Quemado, 30TVL5641 y 30TVL5642, 1150 a 1250 m, matorral de Halimium ocymoides (Herraiz \& Quirós, 11-05-2005, v.v.). Paredes de Buitrago, Puentes Viejas, Las Guijas, 30TVL5640, 1200 m, matorral de H. ocymoides (Quirós, 27-05-2010, v.v.). Paredes de Buitrago, Perímetro de Paredes, 30TVL5241, 1100 m, matorral de Cistus salviifolius y H. ocymoides bajo pinar de P. pinaster procedente de plantación (Herraiz, 11-06-2014, MA 887438), Puebla de la Sierra, Loma del Hocino, 30TVL5839, $1400 \mathrm{~m}$, jaral de C. ladanifer (Quirós \& Herraiz, 05-06-2007, v.v.).

Especie poco citada en la zona norte de la Comunidad de Madrid y que, sin embargo, es abundante y está ampliamente distribuida en los hábitats donde se localizan sus especies huésped. M: Los Molinos, [30TVL00], $1160 \mathrm{~m}$ (Moreno 1984). Madarcos, [30TVL54], 970 y 1030 m (Moreno 1984). Manzanares el Real, La Pedriza, [30TVL21], 1080 m (Moreno 1984). Navacerrada, [30TVL10], 1360 m, en jaral (Moreno 1984). Puebla de la Mujer Muerta [Puebla de la Sierra], [30TVL64] (Cutanda 1861: 592). Gu: Cerro del Charco, [30TVL93], 990 m (Fuente 1985). Sg: Cerezo de Arriba, La Pinilla, 30TVL6061, $1550 \mathrm{~m}$ sobre C. laurifolius (Romero \& Rico 1989).
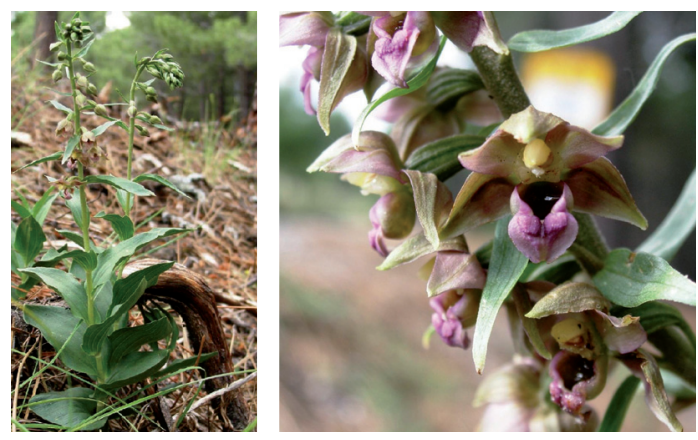

Fig. 3-Epipactis tremolsii Pau. Robledillo de la Jara, Las Vallejas, 30TVL5734 (Á. Quirós, 10-6-2009). 


\section{Cytisus multiflorus (L’Hér.) Sweet}

M: Horcajuelo de la Sierra, 30TVL5444, 1110 m, rodal denso (¿por reproducción vegetativa?) de unos $10 \mathrm{~m}$ de diámetro, en cantuesar-vallicar, atravesado por un camino (Quirós, 21-07-2011, v.v.; Baonza, 2704-2012, MA 886521).

Extraña cita de origen incierto, de este taxón luso-extremadurense. En Madrid citada en el s. XIX de San Martín de Valdeiglesias, [30TUK86] (Cutanda 1861: 253, Colmeiro 1873, Willkomm \& Lange 1874-1880), posiblemente extinta en la actualidad. En el s. XX la única cita es junto a la carretera de Villalba al Puerto de Navacerrada, km 46, 30TVL10, tal vez adventicia e introducida (Benito et al. 2003, Ruiz et al. 1982).

\section{Epipactis tremolsii Pau}

M: Puebla de la Sierra, 30TVL6036, $1110 \mathrm{~m}$, jaral de C. ladanifer (Baonza, 02-08-2011, MA 886537). Puebla de la Sierra, 30TVL6138, $1340 \mathrm{~m}$, brezal de E. arborea (Baonza, 8-09-2011, secos, v.v.). Puentes Viejas, Cuarteles del Riato, 30TVL5638 y 30TVL5639, 1250-1300 $\mathrm{m}$, pedreras de cuarcitas y pinar de Pinus sylvestris y $P$. nigra procedente de repoblación, frecuente (Quirós, 01-VI-2006, v.v.). Robledillo de la Jara, Matachines, 30TVL5733, $1100 \mathrm{~m}$, jaral de C. ladanifer, frecuente (Quirós, 09-VI-2009, v.v.). Robledillo de la Jara, Las Vallejas, 30TVL5734, 30TVL5735 y 30TVL5736, 975 a 1300 m, pinar de $P i-$ nus pinaster procedente de repoblación, dispersa (Quirós, 17-V-2007, v.v.). El Atazar, Cuencas de los Arroyos de Redubia y Refredillo, 30TVL6230, 30TVL6231 y 30TVL6331, de 900 a $1050 \mathrm{~m}$, pinar de $P$. pinaster procedente de repoblación, frecuente (Quirós, 16-V-2007, v.v.).

Especie de la que no hay citas madrileñas (un pliego del SE provincial aparece en anthos.es) aunque a este taxón habría que atribuir las citas de Epipactis helleborine (L.) Crantz. de la Sierra de Ayllón guadalajareña, el SO madrileño y la Dehesa de Arganda (Baonza 2007). Un informe inédito del Cuerpo de Agentes Forestales de la Comunidad de Madrid (2014) sobre las orquídeas madrileñas, además de algunas citas aquí señaladas añade otra para la Dehesa Vieja de Torrelaguna.

\section{Erica scoparia L. subsp. scoparia}

M: La Hiruela, Los Horcajos, 30TVL6246, 1350 m, setos con Rosa spp, escasa (Baonza, 17-09-2011, MA 886533). La Puebla de la Sierra, 30TVL6238, $1200 \mathrm{~m}$, pequeño rodal en zarzal-jaral con Cistus ladanifer (Baonza, 02-08-2011, MA 886532).

Citas que aumentan las referencias previas de La Sierra del Rincón: La Puebla, Dehesa Boyal, 30TVL6338, en robledales con Erica arborea y Cistus ladanifer (Allué \&
Ruíz 1991, López 2001) y Prádena del Rincón, inmediaciones de la Carretera a La Puebla de la Sierra, 30TVL5844, $1350 \mathrm{~m}$ (Allué et al. 1992). Especie frecuente en la vertiente segoviana y guadalajareña de la Sierra de Ayllón (recopilación en Allué et al. 1992, más Cruz et al. 2001, Escarré et al. 1982, Enríquez de Salamanca 2009; Lara et al. 1996, teselas 233, 244 [30TVL67], 1023 [30TVL85], 1188 [30TVL63] en Ruiz de la Torre 1996). En la provincia de Madrid ha sido citado de El Pardo (tesela 348 en López 1996) y antiguamente además de El Escorial, [30TVK09] (Quer, Graells en Cutanda 1861:463, Willkomm \& Lange 1865-1870) y El Paular (Cutanda 1861:463).

\section{Erica tetralix L.}

M: Prádena del Rincón, 30TVL5843, 1430 m, turbera, escasa (Baonza \& Quirós, 02-04-2012, v.v.; Quirós, 01-07-2014, MA 883153).

Especie dispersa por las Sierras de Guadarrama y Ayllón: Gu: La Mortecina, hacia Las Peñuelas, 30TVL6260, 1830-1840 m, turbera de ladera, zona enfangada. (Cruz et al. 2001). M: Por encima de la laguna de Peñalara, 30TVL1921, 2100 m (Fernández 1988). Circo de Hoyocerrado, 30TVL2125, 1790-1850 m (Fernández 1988). Escalerón, arroyo, [30TVL2215], 1650 m (Rivas 1964). Río Manzanares, nacimiento, cuenca alta, [30TVL11], 1650-2100 m (Rivas 1964). Alto Manzanares, La Garganta, 30TVL2112 (Ruiz et al. 1982). Cuerda de los Porrones, La Pedriza de Manzanares [30TVL2229], $1350 \mathrm{~m}$ (Rivas-Martínez et al. 1978). Somosierra, 30TVL5356, 1730-1780 m (Bermejo 2006, datos inéditos). Montejo de la Sierra, [30TVL55] (Mayor, citado en Hernández et al. 1983, aunque no hemos podido encontrar la cita original). A esta especie probablemente corresponda la cita de E. ciliaris de La Maliciosa [30TVL11] (Cutanda 1861: 463), entorno en el que es frecuente en turberas y manantiales. Sg: San Ildefonso o la Granja, Peñalara, 30TVL1824 (García 1995). Torre Val de San Pedro, 30TVL2740 (García 1995). Navafría, 30TVL2941 (García 1995). Aldealengua de Pedraza, El Nevero, 30TVL3038 (García 1995). Prádena, 30TVL4653 (García 1995). Cerezo de Arriba, La Pinilla, 30TVL6060 (Romero \& Rico 1989). Riaza, [30TVL66] (Mayor 1965). San Benito, [30TVL66], 1200$1400 \mathrm{~m}$ (Mayor et al. 1964). Riaza, Ermita de San Benito y de Hontanares, junto a E. vagans (Hernández \& Sáinz 1978).

\section{Genista falcata Brot.}

M: Paredes de Buitrago, Chiviles, 30TVL5540,1130 m, abundante en pequeña vaguada bajo Quercus pyrenaica, en pedrera de cuarcita, ocu- 
pando una banda de unos $600 \times 100 \mathrm{~m}$ de ancho (Herraiz, 12-05-2003, MA 886514; Herraiz, 02-10-2013, MA 886515).

Frecuente en el SO de la provincia de Madrid (Benito et al. 2003), de donde «saltaría» a esta localidad en el NE provincial.

\section{Genista hirsuta Vahl subsp. hirsuta}

M: Paredes de Buitrago-Gandullas, Perímetro de Paredes, 30TVL5138, $1005 \mathrm{~m}$, unas pocas plantas en plantación forestal de Pinus pinaster con Pinus rigida, Cistus laurifolius, Cistus salviifolius, Thymus mastichina, Lavandula stoechas, Halimium viscosum, etc. (Herraiz, 30-82007, MA 886520).

Taxón de clara influencia lusoextremadurense pero que alcanza incluso la provincia de Guadalajara en zonas más meridionales y a menor altitud, entre 700 y $800 \mathrm{~m}$ (Baonza 2001).

\section{Prunus mahaleb $\mathrm{L}$.}

M: Puebla de la Sierra, 30TVL6238, $1150 \mathrm{~m}$, un solo ejemplar arbustivo en pedrera y bajo un viejo Quercus petraea (Baonza, 02-08-2011, v.v.). Cervera de Buitrago, Dehesa Boyal, 30TVL5430, $900 \mathrm{~m}$, un solo ejemplar (comunicación de los Agentes Forestales de la zona). Robledillo de la Jara, El Carrascalejo, 30TVL5431, unos veinte ejemplares arbustivos y algunos pies más jóvenes, dispersos en linderos de piedra en seco, con encinas, quejigos, arce de Montpellier, enebro de la miera, cornicabra y majuelos (Quirós \& Cezón, 02-04-2009, v.v.; Quirós, 14-05-2014, MA 883151). Puentes Viejas (Serrada-Paredes de Buitrago), Peña del Águila, 30TVL5640, más de veinte ejemplares, jóvenes en su mayoría, en canchal de cuarcita, con enebro común, sabina, guillomo y mostajo (Herraiz, 15-09-2003, v.v.).

En Madrid señalado antiguamente de Guadarrama con interrogación (Cutanda 1861: 273) y más recientemente de la garganta del Arroyo Aguilón en Rascafría, 30TVL2722, 1460 m (Revilla 1995, Enciso 2008) y del límite con la provincia de Guadalajara, en el Río Jarama, a 1280 y $1250 \mathrm{~m}, 30$ TVL5851 y30TVL5949 (Lara et al. 1996:244). Alguna de las citas precisan la presencia en la Tesela 1382 [30TVL53] (Ruiz 1996).

Pterospartum tridentatum subsp. lasianthum (Spach) Talavera \& P. E. Gibbs

M: La Hiruela, Cerro Salinero, ladera SO y SE, 30TVL6145, rodales a 1610-1550 m respectivamente, el primero escaso, con unas 20 plantas en brezal de Calluna vulgaris (Baonza, 13-10-2011, v.v.) y el segundo en brezales y jarales mixtos (Quirós 2010, v.v.). La Hiruela, valle del Arro- yo Salinero, 30TVL6245, laderas orientada hacia el (SE)S (SW) y N-NE, entre 1300-1400 m, en brezal mixto o jaral de jara pringosa y jara estepa con algunos rodales donde es dominante, sobre esquistos y pizarras (Baonza \& Quirós, 24-05-2014, MA 886519). Gu: Colmenar de la Sierra, 30TVL6245, 1250 m, colindante con la cita anterior, en orientación NO.

Confirma la persistencia en La Hiruela de la especie, recolectada por Isern en 1858 (Cutanda 1861, Colmeiro 1873, Ruiz et al. 1982, Benito et al. 2003). En la provincia de Madrid también fue recolectada en el Paular en el s. XIX (Benito et al. 2003) y existen citas antiguas de Somosierra (Cutanda 1861:252; confirmadas 7 plantas muy cerca en Robregordo, 30TVL5051, junto a la antigua N1, Bermejo 2006, datos inéditos). En Guadalajara y en Segovia es citada escasa de la Sierra de Ayllón (Cutanda 1861: 252, Mayor 1965, 1975; Rivas 1964, 1979; Fuente 1985; Romero \& Rico 1989; Allué et al. 1992). Teselas 478 [30TVL66], 528 [30TVL85], 526 [30TVL86], 1373 [Lozoyuela, 30TVL43, probable errata, tal vez se refiera a Genista cinerascens o G. florida, especies no señaladas pero presentes en la tesela] (Ruiz 1996).

\section{Pyrus bourgaeana Decne}

M: Madarcos-Prádena del Rincón, 30TVL5343, 1080 m, pequeño rodal de rebrote (Baonza, 09-07-2011, v.v.). Prádena del Rincón, 30TVL6241, 1185 m, matorral de $H$. ocymoides, 1 ejemplar pequeño (Baonza, 06-09-2011, v.v.). Paredes de Buitrago (término municipal de Puentes Viejas), Prado Concejo, 30TVL5440, 1060 m, rodal con bastantes ejemplares algunos de tamaño casi arbolillo, protegidos hacia 2004 con un cercado por los agentes forestales (Herraiz, 2004, v.v.). Paredes de Buitrago (término municipal de Puentes Viejas), ladera de Peña Águila, 30TVL5740, 1420 m, un ejemplar de gran tamaño, $40 \mathrm{~cm}$ diámetro en la base, en pedrera cerca de una sabina albar de gran tamaño (Herraiz, v.v.). Paredes de Buitrago (término municipal de Puentes Viejas), 30TVL5240, $1020 \mathrm{~m}$, algunos ejemplares sueltos a los lados de una calleja (Herraiz, 16-4-2003, MA 887437).

Especie propia del 0 peninsular de clima mediterráneo, penetra hacia el este por la rampa de la Sierra de Guadarrama y zonas adyacentes de la Fosa del Tajo hasta la provincia de Guadalajara (Baonza 2001, más adicciones en Gabriel y Galán (2005) y Enciso (2008), alcanzando incluso el NE de Guadalajara en Anguita, 30TWL4938, a 1150 m, Baonza 1009-2010, v.v.). Las citas señaladas, junto a las de la Dehesa Boyal de Madarcos, 30TVL5244, 1100 m (Bermejo 2006, datos inéditos) son las más septentrionales en Madrid.

\section{Xiphion latifolium Mill.}

M: Montejo de la Sierra, Dehesa boyal, 30TVL5546, 5646 y 5647, 1200-1240 m, melojar (Quirós, 18-06-2011, v.v.) y La Retuerta, 
30TVL5746, $1200 \mathrm{~m}$, melojar fresneda en parcelas cercadas con muros de piedra seca (Quirós, 04-07-2011, v.v.). Prádena del Rincón, dehesa de Lomo Peral, 30TVL5543, $1100 \mathrm{~m}$, melojar y Los Cotorros, 30TVL5643, 1100 m, melojar fresneda (Quirós, 14-06-2009, v.v.). La Hiruela, Dehesa boyal, 30TVL6247 y 30TVL6347, 1200-1350 m, melojar y claros en melojar, localmente abundante (Quirós, 22-06-2005, v.v.; Quirós, 2-06-2014, MA 883152).

Se aumenta el conocimiento de esta interesante especie, muy poco citada en la zona. M: Dehesa de Somosierra, 30TVL5152, 5153, 5252, 5253 y 5154 (Cebolla et al. 1994, Romero \& Rico 1989); Montejo de la Sierra: prado Nazaret, 30TVL5745 (D. Galán, 06-2011, MA 849786) y riberas del Jarama cerca del Arroyo del Paso Malo [30TVL5852] (Bellot 1944, localidad donde no se ha vuelto a citar). Robregordo y La Acebeda (Bermejo 2006, datos inéditos). Gu: El Cardoso de la Sierra, 30TVL6050 y 30TVL6349 (D. Galán 06-2010, MA 819286 y MA 819282).

\section{DISCUSIÓN}

De las citas de interés señaladas, cabe resaltar las especies más frecuentes en el 0 peninsular, que denotan influencia atlántica, a pesar de la posición central de la comarca, y que a veces son más frecuentes aquí que en la Sierra de Guadarrama, más occidental. A este conjunto pertenecen Allium scorzonerifolium, Adenocarpus argyrophyllus, Chaetonychia cymosa, Cytisus multiflorus, Erica tetralix; Erica scoparia subsp. scoparia, Genista falcata, G. hirsuta, Pterospartum tridentatum subsp. lasianthum y Pyrus bourgeana. Esta influencia atlántica queda reforzada con las citas en esta comarca 0 la Sierra de Ayllón en su conjunto de: Lobelia urens (Baonza 2012); Erica australis, Osmunda regalis (revisadas en Baonza et al. 2013); Erica vagans (Hernández \& Sainz 1984); Genista anglica (revisado por Allué et al. 1992, a las que se puede añadir en el área de Riaza observaciones propias en San Benito, 30TVL6063, a 1360 m junto a E. vagans); Alnus glutinosa, formando las alisedas hercínicas (Lara et al. 2004) y Halimiun ocymoides (revisado en Baonza 2000).

De la importancia biogeográfica de esta influencia atlántica da idea que los matorrales dominados por Erica australis y Halimiun ocymoides se han utilizado para establecer el límite biogeográfico entre los (sub)sectores ayllonense y guadarramense, situado en plena Sierra de Guadarrama en el valle de la Acebeda y el $\mathrm{N}$ de Lozoyuela (Fernández 1991), al 0 de la zona aquí estudiada. Y esto a pesar de que en principio la principal diferencia señala- da entre ambos (sub)sectores era la mayor influencia florística de óptimo septentrional en la zona ayllonense, con comunidades características como los hayedos y melojares ombrófilos ibérico-ayllonenses o la presencia de flora centroeuropea como Erica cinerea, Galium odoratum, Paris quadrifolia, Quercus petraea, etc., además de la presencia los brezales rojos, la ausencia de verdaderos pinares oromediterráneos naturales o la sustitución de los piornales de Cytisus purgans por brezales (Fernández 1991, Rivas et al. 1990, Mayor 1965b, Hernández \& Sainz 1984: 62-63, etc.).

Esta diferenciación entre la zona ayllonense de la guadarramense se ha atribuido a un gradiente climático E-O en el Sistema Central, con mayores precipitaciones estivales en la zona ayllonense (Fernández 1991, Rivas et al. 1990). Sin embargo, realmente existe gran variabilidad climática según la altitud, latitud y longitud geográfica y así, por ejemplo, en la vertiente sur este efecto aparece menos claro, notándose más el descenso de las precipitaciones anuales de oeste a este (Gavilán et al. 1998).

Esta variabilidad climática genera un gran mosaicismo en la distribución de la flora y vegetación según la orientación de las laderas (Hernández \& Sainz 1984: 62), lo que causa que no se puedan definir límites biogeográficos claros basados en este criterio. La distribución de las plantas o comunidades supuestamente diagnósticas tampoco se circunscribe a uno u otro sector. Hay citas históricas de hayas en la Sierra de Guadarrama, destacando la de Casiano de Prado en «Montejo, Somosierra, en el Paular y en Cercedilla, que eran antes más comunes a uno y otro lado de la sierra, y de ellas se hacían cortas hasta mediados del siglo pasado. En la actualidad están a punto de desaparecer del todo si no se procura su reproducción» (Prado 1864: 34). Otras muchas especies de las señaladas como centroeuropeas (Quercus petraea, Paris cuadrifolia, etc) alcanzan la Sierra de Guadarrama (véase por ejemplo Baonza et al. 2013); los robledales guadarrámicos del Luzulo forsteri-Quercetum pyrenaica son dominantes en gran parte de la zona ayllonense (Fuente 1985, Cruz \& Peinado 1996), donde sí existen pinares naturales (Cruz \& Peinado 1996); los matorrales de H. ocymoides penetran en Guadarrama a favor de la Cañada Real Segoviana, probablemente por dispersión del ganado trashumante (Baonza 2000); y la presencia de comunidades xerófilas como el sabinar de Tamajón (en suelo calizo y silíceo) 0 la abundancia de jarales de Cistus ladanifer y/o C. laurifolius (Hernández \& Sainz 1984: 63, Fuente 1985, Cruz \& Peinado 1996, López 2001) no hablan de una vegetación claramente más ombrófila en el sector ayllonense que en el guadarramense. 
Lo que sí es evidente en la Sierra del Rincón y que se puede extender a toda la zona ayllonense, es la marcada diferencia que se produce en los matorrales por el cambio del sustrato geológico entre los gneises del macizo de Somosierra y los esquistos, pizarras y cuarcitas de la Sierra de Ayllón. Sobre el gneis predominan matorrales de genisteas, principalmente piornales de Cytisus oromediterraneus y escobonales de Genista cinerascens, ambos frecuentemente con Adenocarpus hispanicus (a veces dominante) y puntualmente con Erica arborea. Son muy abundantes en el macizo de Somosierra y se extienden por la Sierra de Guadarrama. Sin embargo, sobre los esquistos, pizarras y cuarcitas se instalan de forma general brezales más o menos mixtos (Erica arborea, Erica australis, Calluna vulgaris y en algún caso Erica scoparia) salvo en las solanas medias y bajas de la Sierra de La Puebla donde dominan los jarales. Los brezales alcanzan las cumbres más altas (La Tornera, 1866 m; Peña de la Cabra, 1831 $\mathrm{m})$, constituidos principalmente por $E$. arborea, sin que aparezcan piornos ni piornales.

La ausencia de piornos y piornales de $C$. oromediterraneus también ha sido señalada en las sierras ayllonenses de Guadalajara (Cruz \& Peinado 1996, Fuente 1985), quedando las manifestaciones más orientales de esta es- pecie en el Sistema Central, en el collado de San Benito, próximo al puerto de la Quesera (Hernández \& Sainz 1984: 70) que marca el límite, no siempre reconocido, entre el macizo de Somosierra y la Sierra de de Ayllón en sentido estricto. Genista cinerascens también es muy rara en la zona ayllonense (Cruz \& Peinado 1996, Fuente 1985), sin estar citada de la vertiente segoviana (Romero \& Rico 1989), al contrario de lo que ocurre en Somosierra y Guadarrama, donde forma frecuentemente escobonales en el piso supramediterráneo y oromediterráneo inferior. Las únicas genisteas de estas zonas de pizarra y cuarcita del Madrid ayllonense son Genista florida y Cytisus scoparius (frecuentes como ocurre en todo el Sistema Central), la presencia puntual de Cytisus striatus (Baonza et al. 2013) y, lo más particular, las poblaciones de Adenocarpus argyrophyllus y Pterospartum tridentatum aquí señaladas, ausentes o rarísimas en la zona de Somosierra o de la Sierra de Guadarrama.

\section{AGRADECIMIENTOS}

Miguel Ángel Bermejo Roa nos facilitó su trabajo inédito sobre flora vascular de interés de la comarca de Buitrago (2006).

\section{BIBLIOGRAFÍA}

Allué, M. \& Ruiz del CASTillo, J. 1991. Fragmenta chorologica occidentalia, 3614-3623. Anales Jard. Bot. Madrid 49(1): 123-124.

Allué, M.; García-López, J. M.; Ruiz Del Castillo, J.; Ruiz De La Torre, J. \& Martínez-Labarga, J. M. 1992. Notas sobre flora y vegetación en el sector oriental del Sistema Central. Ecología 6: 51-65.

BAONZA, J. 2014. Corrección de la cita de Sorbus intermedia de Montejo: S. hybrida L. en Madrid. Bot. Complut. 38: 191.

BAONZA, J. 2012. Citas de flora vascular de interés de la Sierra del Rincón (Madrid, España). Bot. Complut. 36: 105-111.

BAONZA, J. 2007. Algunas plantas raras en Madrid. Nuevas citas y revisión corológica. Bot. Complut. 31: 87-95.

BAONZA, J. 2001. Influencia luso-extremadurense en la mitad oriental de la provincia de Madrid. Ecología 15: 101-109.

BAonZA, J. 2000. Poblaciones de Halimium ocymoides disyuntas de su principal área de distribución madrileña: ¿un caso de dispersión dirigida por las cañadas? Ecología 14: 253-259.

Baonza Díaz, J.; Martínez-García, F.; Bernal González, R.; Durán Gómez, J. A. \& Blanco Castro, E. 2013. Notas florísticas de la Sierra de Guadarrama (Madrid, Segovia). Ecología 25: 137-174.

BeLLOT, F. 1944. Estudio sobre la vegetación y la flora de la comarca de Somosierra. Anales R. Acad. Farmacia 10: 109-130.
Benito, M.; De Mendoza, G. \& Moreno, L. 2003. Recopilación corológica de la tribu Cytisea Bercht. \& J. Presl. (Papilionoideae, Leguminosae) en la Comunidad de Madrid. Bot. Complut. 27: 119-135.

Cebolla, C. \& Rivas, M. A. 1994. Atlas florae matritensis (Amaryllidaceae, Iridaceae, Liliaceae, Orchidaceae). Fontqueria 41: 1-206.

Colmeiro, M. 1873. Genisteas y Antillideas de España y Portugal. Anales Soc. Esp. Hist. Nat. 1(3): 289-378.

Cruz Rot, M. de la \& Peinado Lorca, M. 1996. El paisaje vegetal de la cuenca del río Henares (I): comunidades arbóreas y arbustivas. Wad-al-Hayara: Revista de estudios de Guadalajara 23: 335-396.

Cruz Rot, M. de la; Rejos Ballesteros, F. J.; Pavón García, J.; ÁldVarez Jiménez, J. \& Bartolomé Esteban, C. 2001. Estudio sobre la situación y problemática de conservación de las especies de flora y comunidades de vegetación de los pisos bioclimáticos oro y crioromediterráneos de la Sierra de Ayllón (Guadalajara). Ed. Departamento de Biología Vegetal. ETSI Agrónomos, Universidad Politécnica de Madrid. (Citas en anthos.es).

Cuevas, J. A.; De las Heras, P.; López, N. \& Roldán, M. J. 2005. Caracterización paisajistica y ecológica de la Sierra del Rincón (Madrid). Centro de Investigaciones Ambientales de la Comunidad de Madrid. 
CutAnda, V. 1861. Flora compendiada de Madrid y su provincia. Imprenta Nacional, Madrid.

Enciso Encinas, E. 2008. Fuentes semilleras de la Comunidad de Madrid, tomo II, Consejería Medio Ambiente y Ordenación del Territorio, Madrid.

Enríquez de Salamanca SÁnchez-Cámara, A. 2009. Adiciones y correcciones a la flora de la cuenca alta del río Jarama (Guadalajara y Madrid). Flora Montiberica 42: 55-64.

Escarré, A.; Bellot, J.; Lledó, M. J.; Hervás, J.; Pujalte, E. $\&$ GARRIDO, P. 1982. Balance de nutrientes en pequeñas cuencas de encinar. I. Selección de la zona de estudio e instalación de instrumentos para la recogida de datos. Mediterránea, Ser. Biol. 6: 141-165.

Fuente, V. DE LA 1985. Vegetación orófila del occidente de la provincia de Guadalajara (España). Lazaroa 8: 123-219.

Gabriel y GalÁn, J. M. 2005. Notas florísticas sobre plantas del norte de España. BIOCIENCIAS, Revista de la Facultad de Ciencias de la Salud 3: 3-11.

GARCía ADÁ, R. 1995. Estudio de la flora y vegetación de las cuencas alta y media de los ríos Eresma, Pirón y Cega (Segovia). Tesis de Licenciatura. Facultad de Biología, Universidad Complutense.

Gavilán, R.; Fernández-González, F. \& Blasi, C. 1998. Climatic classification and ordination of the Spanish Sistema Central: relationships with potential vegetation. Plant Eco$\log y$ 139: 1-11.

Hernández Bermejo, J. E. \& Sainz Ollero, H. 1984. Ecología de los hayedos meridionales ibéricos: el macizo de Ayllón. $2^{\mathrm{a}}$ ed. IEAPA. Madrid.

IbÁÑEZ, N.; Soriano, I. \& MontSERrat, J. M. 2009. L'herbari Bernades a l'Institut Botànic de Barcelona (BC). Collect. Bot. (Barcelona) 28: 31-63.

Lara, F.; Garilleti, R. \& Calleja, J. A. 2004. La vegetación de ribera de la mitad norte española. CEDEX, Madrid.

Lara, F.; Garilleti, R. \& Ramírez, P. 1996. Estudio de la vegetación de los ríos carpetanos de la cuenca del Jarama. CEDEX, Madrid.

López LeIVA, C. 1996. V Relación numerada de contenido de teselas. En J. Ruiz de la Torre (Dir.), Mapa Forestal de España E 1:200.000. Madrid, hoja 5-6: 121-252. Ministerio de Medio Ambiente.

LóPEz EstÉBANEZ, N. 2001. Usos y organización espacial de la vegetación en el valle de La Puebla (Madrid). Eria 56: 235-253.

MaYOR, M. 1965a. Estudio de la flora y vegetación de las sierras de Pela, Ayllón y Somosierra. Tesis Doctoral, Universidad Complutense, Madrid.

Mayor, M. 1965b. Especies pirenaicas en el tramo oriental del Sistema Central. Anales Inst. Bot. Cavanilles 22: 407-420.
Mayor, M. 1975. Datos florísticos sobre la Cordillera Central (Somosierra, Ayllón y Pela). Anales Inst. Bot. Cavanilles 32(2): 323-347.

Mayor, M.; Díaz, T. E.; NAvarro, F.; Martínez, G. \& AndréS, J. 1974. Los pastizales del Sistema Central. Nota I: Somosierra, Ayllón y Pela. Rev. Fac. Ci. Univ. Oviedo 16: 283-322.

Moreno, J. M. 1984. Estudio ecológico comparado de los jarales de Madrid, I. Variación anual de la humedad del suelo. Lazaroa 6: 105-126.

Moreno Saiz, J. C. \& Saiz Ollero, H. 1992. Atlas corológico de las monocotiledóneas endémicas de la Península Ibérica e Islas Baleares. ICONA. Madrid

Muñoz Jiménez, J. \& Sanz Herraiz, C. 1995. Guía física de España, 5. Las montañas. Alianza Editorial.

PRADO, C. DE. 1864. Descripción física y geológica de la provincia de Madrid. (Reproducción facsímil 1998. Inst. Tec. Geominero de España).

ReviLla, A. 1995. Biología, distribución y cultivo del cerezo de Santa Lucía. Quercus 116: 38-43.

Rivas Goday, S. 1958. Nuevos órdenes y alianzas de Helianthemetea annua Br.-Bl. Anales Inst. Bot. Cavanilles 15: 539-651.

Rivas Martínez, S. 1964. Estudio de la vegetación y flora de las Sierras de Guadarrama y Gredos. Anales Inst. Bot. Cavanilles 21(1): 5-325.

Rivas Martínez, S. 1979. Brezales y jarales de Europa occidental. Lazaroa 1: 1-127.

Rivas Martínez, S.; Crespo, A.; Cubas, P. \& Moreno, J. M. 1978. Lycopodiella inundata (L.) Holub en la Sierra de Guadarrama (España). Anales Inst. Bot. Cavanilles 34(2): 535 537.

Rivas Martínez, S.; Fernández GonzÁlez, F.; SÁnchez-MATA, D. \& Pizarro, J. 1990. Vegetación de la Sierra de Guadarrama. Itinera Geobot. 4: 3-132.

Romero, T. \& Rico, E. 1989. Flora de la cuenca del río Duratón. Ruizia 8: 7-438.

Ruiz de la Torre, J. 1996. Mapa Forestal de España E 1:200.000. Segovia, hoja 5-5. Ministerio de Medio Ambiente, Madrid.

Ruiz, J. (Dir.); Abajo A.; Carmona E.; Escribano R.; Ortega C.; Rodríguez, A. \& Ruiz, J. 1982. Aproximación al catálogo de plantas vasculares de la provincia de Madrid. Consejería de Agricultura y Ganadería, Madrid.

Vera, J. A. (Ed.). 2001. Geología de España. Soc. Geol. de España y Inst. Geol. y Minero de España.

Willkomm, M. \& Lange, J. 1874-1880. Prodromus florae Hispanicae, 1-3. Stuttgart. 